Relations industrielles

Industrial Relations

\title{
Management Operations Research, Norbert Lloyd Enrick, Holt, Rinehart \& Winston, Inc., 833 Oxford St., Toronto 18, Ont. 1965. 320 pages.
}

\section{Bertrand Belzile}

\section{Volume 21, numéro 2, 1966}

URI : https://id.erudit.org/iderudit/027693ar

DOI : https://doi.org/10.7202/027693ar

Aller au sommaire du numéro

Éditeur(s)

Département des relations industrielles de l'Université Laval

ISSN

0034-379X (imprimé)

1703-8138 (numérique)

Découvrir la revue

Citer ce compte rendu

Belzile, B. (1966). Compte rendu de [Management Operations Research, Norbert Lloyd Enrick, Holt, Rinehart \& Winston, Inc., 833 Oxford St., Toronto 18, Ont. 1965. 320 pages.] Relations industrielles / Industrial Relations, 21(2), 307-307. https://doi.org/10.7202/027693ar

Tous droits réservés @ Département des relations industrielles de l'Université Laval, 1966
Ce document est protégé par la loi sur le droit d'auteur. L’utilisation des services d'Érudit (y compris la reproduction) est assujettie à sa politique d'utilisation que vous pouvez consulter en ligne.

https://apropos.erudit.org/fr/usagers/politique-dutilisation/ 
Canada. On pourra peut-être le chicaner sur des questions de détails, mais l'ensemble est sérieux et très imposant.

Nous espérons que cet appel à la conscience des responsabilités tant chez les Canadiens que chez les Européens trouvero un écho.

\section{Gérard Dion}

Monagement Operations Research, Norbert Lloyd Enrick, Holt., Rinehart $\mathcal{E}$ Winston, Inc., 833 Oxford St., Toronto 18, Ont. 1965. 320 pages.

Qu'ils le veuillent ou pas, les dirigeants de l'entreprise devront avoir de plus en plus recours à la recherche opérationnelle. En effet cette méthode scientifique s'inscrit dans la méthodologie vers une science de l'action et les dirigeants, qui sont avant tout des hommes d'action qui prennent continuellement des décisions dans leur entreprise, devront faire appel da cette méthode des modèles mathématiques qui permet précisément de mieux préparer les décisions.

II ne faut quand même pas penser que la recherche opérationnelle peut se substituer complètement aux dirigeants. Certes les ordinateurs prennent plusieurs décisions en vertu des programmes préalablement établis par les dirigeants, mais ces derniers auront toujours à prendre de nom- breuses décisions et surtout les plus difficiles.

C'est donc à juste titre que dans la première partie de son volume, l'auteur définit le rôle de la recherche opérationnelle dans l'administration moderne et traite des méthodes comptables qui $y$ sont utilisées; il soulève également le problème épineux de la prise de décision face à l'incertitude.

Depuis quelques années, la littérature compte de nombreux ouvrages sur l'utilisation de la recherche opérationnelle dans la gestion des entreprises. Le contenu de tels ouvrages varie toutefois assez fortement les uns des autres. La distinction porte à la fois sur les problèmes étudiés et sur les modèles mathématiques présentés. $M$. Enrick, pour so part, s'attarde à quelques techniques importantes: dons la deuxième partie, il expose la programmation linéaire et la méthode du chemin critique; dans la troisième, il présente le modèle des phénomènes d'attente dans son utilisation pour la gestion des inventaires; et dans la dernière partie, l'auteur discute de l'échantillonnage et de l'analyse statistique.

Les faibles prérequis en mathématiques rend cet excellent volume accessible aux étudiants en administration de l'entreprise et à la plupart de nos chefs d'entreprises. Nous le conseillons à tous les intéressés.

Bertrand Belzile

\section{PUBLICATIONS RÉCENTES}

\section{GENERALITES}

«Revolucion, reforma y conservatismo: tipos de politicas agrarias en Latinoamerica $\$$, Oscar Delgado, Revista Brasileira de Ciencias Sociais, Julho 1963, Vol. III, Num. 2, pp. 172-252.

«Le deuxième congrès mondial de la population. 》, Belgrade, 30 août au 10 septembre 1965, en collaboration, Population, 20e année, no 6, novembre-décembre 1965, pp. $941-1186$.

«L'histoire de la population et de la structure sociale », par Peter Laslett, Revue internationale des sciences sociales, $\mathrm{Vol}$. XVII, (1965), no 4, pp. 626-640.
«Mutations de la propriété 》, André Tunc, Guy Houist, Louis Estrangin, André Piette, numéro spécial de Recherche sociale, no 2, novembre-décembre 1965, pp. 1-48.

«Popperism: The Scarcity of Reason 》, by James Petras, Science $\&$ Society, Vol. XXX, No. 1, Winter, 1966, pp. 1-11.

«An Armour Automation Report», by Eaton $H$. Conant, Monthly Labor Review, Vol. 88, No. 11, November 1965, pp. 12971302.

«Considérations sur l'évolution actuelle du capitalisme 》, LuC Bourcier de Carbon, Droit Social, no 1, janvier 1966, pp. 1-15. 